\title{
Society for Pediatric Research - 2007 Presidential Address: Expanding the Research Continuum - From Bench to Implementation
}

\author{
GARY L. FREED \\ Child Health Evaluation and Research (CHEAR) Unit, Division of General Pediatrics, University of Michigan, Ann Arbor, MI 48109
}

$\mathrm{I}_{\mathrm{s}}^{\mathrm{t}}$ t has been an honor to have served as the president of the SPR over the past year. As the first president with a focus on health services research, there has been a bit of a culture shock for me and for the organization-fortunately, mostly in a positive way.

My remarks today will focus on the new directions in which pediatric research is heading and the way in which we all can, and I posit should, be thinking about how to work together for the good of children as we move forward. Because I harbor no illusions regarding the fact that I am only one voice in the pediatric research community, and my remarks today will call for a departure from "business as usual" in our research enterprise, I have asked 2 distinguished members of our community to reflect on the ideas I will present to you today and provide their views on the future of pediatric research: Bruder Stapleton, Chair of the Department of Pediatrics at the University of Washington, and immediate past chair of AMSPDC, the Organization of Pediatric Department Chairs, and Duane Alexander, the Director of the NICHD.

My topic this morning is "Expanding the Research Continuum: Taking us from Bench to Bedside, to Bench to Implementation.

I would first like to lay out for you an illustration of how I view our current research paradigm for translational research, or what we call "bench to bedside" (Fig. 1). The process starts with basic science discovery and moves to animal studies and then to controlled clinical trials in humans. Again, this is what we call "bench to bedside". However, when we stop the research continuum at this point, we are leaving off the critical phases that bring these results from the in vitro clinical trial to the in vivo world of clinical practice.

There is, in fact, another phase of the research continuum that picks up after the clinical trial ends and is summarized under the heading of health services research. This phase includes studies of the effectiveness and cost of interventions; studies related to access and disparities of care; the organiza-

Received May 16, 2007; accepted May 20, 2007.

Correspondence: Gary L. Freed, M.D., University of Michigan, 300 North Ingalls Building 6E08, Ann Arbor, MI 48109-0456; e-mail: gfreed@med.umich.edu

Presented at the 2007 Annual Meeting of the Pediatric Academic Societies, Toronto, Ontario, Canada. tion, financing and delivery of care including studies of the influences of both physician behavior and patient behavior on the adoption of clinical interventions and finally, the ultimate short and long term health outcomes. I will present arguments to you this morning that there is an imperative for the investigators comprising these two phases of research to actively work together and form teams to address both the advancement of science and the most pressing issues facing child health today ... that we should expand our thinking of research from bench to bedside, to bench to implementation.

But first, a disclaimer of some important realities: I realize what I will present will not be a fit for every investigatorthere are no absolutes. I recognize that some very important basic work does not, and will not, lead to specific applications, and that not all research follows the simplistic linear path I just showed you. And as we all know, not everyone can (or even should) work in teams.

I also recognize that we have achieved much from our current paradigm of bench to bedside. There has been both good science and medical advances. But, there is also a frequent disconnect between science and application that results in proven interventions that will never be used or widely implemented. These are truly opportunities lost-perhaps in some instances, even wasted research effort if there is no, or failed implementation. The disconnect between clinical and health services researchers has also, as a recent JAMA article demonstrated, led to an increased rate of contradicted clinical and health services research studies. We end up with our share of market successes but also our share of market failures for our research ideas and products where implementation is playing catch-up to new and exciting developments or where implementation never happens at all. The bottom line is we can do better.

Our pattern of not working together also has resulted in some rather destructive characteristics of our current paradigm, some of which I believe are summarized in the term "tribalism." Especially in the current funding climate, it has led many of us to a sort of "survivor complex" where we perceive the environment as "us versus them." This can, and does, take many forms. It could be generalists versus specialists, or bench versus clinical versus health services research- 
Biomedical

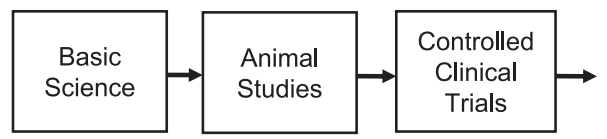

Health Services Research

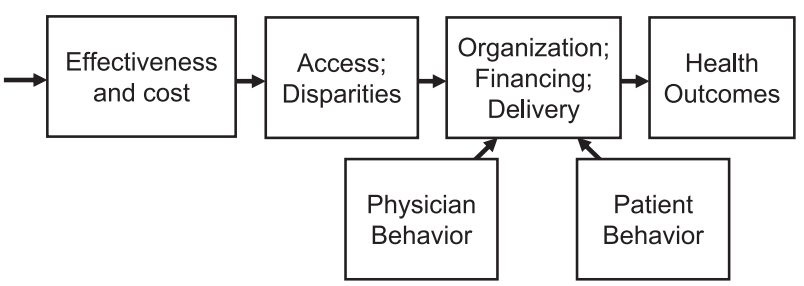

Figure 1. Translational Research: Two Phases of the Research Continuum.

ers. No matter which grouping we fall into, we often feel that although we are more important, or that we are the real scientists, they - whomever they may be - get all of the funding, all of the attention or all of the credit.

I realize I have only spoken in generalities to this point, and many of you are wondering if I can point to specific examples of where our current paradigm has not served us well. I'd now like to share 3 specific examples and hope they spur you to think of others on your own. My three are: 1) the withdrawal of the Lyme disease vaccine from clinical use, 2) the delayed implementation of the Hib vaccine, and 3) the recent implementation of expanded newborn screening.

Back in the late 1990s, at least partially in response to public concern and media attention to Lyme disease, a new vaccine for its prevention was developed, licensed and released with much fanfare. However, the vaccine was never widely used and, in a relatively short amount of time, was removed from the market.

It is not a secret that there were tremendous expenditures to develop this vaccine; tens of millions of dollars from the government, including the National Institutes of Health, and private sources. There were many basic and clinical research studies conducted. And, unfortunately, numerous careers devoted, and perhaps even some wasted, developing an intervention that will not be used. Scarce resources, both intellectual and financial, for children's health that cannot be recovered.

So what went wrong? Although a full discussion of this issue could occupy an entire presentation, I have simplified this to 3 unforeseen problems. First, although there was a general perception the vaccine would be well received, there were not rigorous studies conducted to assess potential demand from either providers or patients-such studies could have raised a danger signal early in the process to those conducting the basic or clinical research. Second, the vaccine came out at a time when there were several other newly available vaccines, perhaps seen by those same providers and patients as more important, thus resulting in a "crowd out" phenomenon for this vaccine. There was no coordination between those conducting the clinical trials and those who might have conducted the implementation research to understand this hurdle that the vaccine might face. And finally, the safety issues raised for this vaccine were not fully assessed and appreciated, and subsequently not addressed effectively in either the provider or patient arenas.

Although impossible to predict, I believe that better coordination of research agendas among different types of researchers would have led to either a redirection of research earlier in the process and thus allowing the human and financial research resources to have been better used, or to an improved likelihood of adoption following the clinical trial.

Next, let's look at the delayed implementation of the Hib vaccine when the recommendation was changed from 15 mo of age to 2 mo of age, back in the early 1990s, based on then newly available clinical trial data. This change in recommendation represented a tremendous opportunity to prevent additional Hib disease. Unfortunately, there was a limited dissemination strategy for the new recommendation and minimal, if any, preparation of physicians in practice for this change. There was a lack of coordination of the research agendas between those conducting the clinical trials and those who conduct implementation research. This led to widespread adoption of the new recommendation taking over 12 mo to occur and resulted in many preventable cases of Hib meningitis causing either death or severe chronic sequelae. Many of these cases simply did not have to occur. Lives lost or permanently damaged as a result of poor coordination across the research continuum.

Next, let's look at a more current example, that of expanded newborn screening with tandem mass spectrometry. Over the past several years, there has been a dramatic increase, in almost every state, of the number of newborn screening tests conducted due to the development and utilization of tandem mass spectrometry, a relatively low cost method of screening for multiple disorders.

Unfortunately, the utilization of this new technology was done with little or no preparation of the health care system to adjust to these changes. As a result, physicians in practice began to be notified their patients had screened positive for, at worst, some diseases of which they had never heard, or, at best, diseases for which they were unfamiliar. Some state screening programs still do not have processes in place to notify primary care providers of positive screens in a timely or accurate fashion. Responsibility for payment for follow up of positive screens for new additions to the screening programs is still in flux. Parents, already unfamiliar with the tests in the previous iterations of newborn screening, are unsure what to make of the new tests. Entire systems are struggling to make sense of a plethora of false positive results for a host of new diseases, and physicians are struggling to prevent unnecessary cases of vulnerable child syndrome. Better coordination of those who developed these tests with those who conduct research on the implementation of new technology would have resulted in a smoother and more effective execution of their integration into practice.

So, some of you, I am sure, remain unconvinced about why there is an imperative to change the existing paradigm. There is an undeniable increase in the rapidity of the development of new technologies. The time from concept to intervention is shrinking. We must begin to think about implementation almost co-incident to basic research endeavors. Also, there are 
increasing cost pressures in health care. Again, we must make sure that in this ever shrinking pool of research resources that every dollar spent, is a dollar well spent.

We would also be naïve if we did not say out loud that the changing demographic nature of the US, the aging of America, is resulting in both public and private capital being increasingly focused on the elderly. We must make the most of the resources allocated to children and ensure that they derive maximal benefit from our pathways of new discoveries. We simply cannot afford additional failures to implement.

And, as Dr. Alexander will speak about, the National Institutes of Health Roadmap initiatives further encourage us to think about a bench to implementation paradigm.

There is also a recent change in the inclusiveness of the Pediatric Scientist Development Program, one of the premier scientific training programs in our field. Clinical and health services research, in addition to basic work, is now being actively encouraged in the application process and, I have been assured, guaranteed a level playing field in the competition. This will facilitate a larger pool of rigorously trained researchers across the research continuum.

We must also be responsive to the demands of the marketplace in an ever focused "results oriented" society. Applied science, with an implementation perspective to benefit children, will have a greater likelihood of attracting funding in the future.

Finally, and most importantly, changing the paradigm to bench to implementation will give us all a greater likelihood of decreasing the morbidity and mortality affecting children.

There is also a business case for changing our current paradigm. It is no surprise to any of us that there is limited funding for pediatric research. This again means that every dollar spent must be a dollar well spent. And, as we increasingly say in the Detroit metropolitan area, getting any car to the showroom is not enough; we also need to know better which cars to build. This will only occur with feedback from implementation researchers to our basic science colleagues. We also can recognize that product (in our case, research) placement, pricing and packaging are keys to successful implementation. And finally, success in changing clinical outcomes, whether for rare or common diseases, will breed more success - and more research funding as a result.

So-hopefully you are now thinking that there is some merit to this idea, but you are wondering, and rightfully so, what is in it for me? Well, for all types of investigators, several things. First, and most importantly, there is an increased potential to make a difference in the lives of children. Second, - as you will hear from Dr. Alexander shortly, more competitive applications to the National Institutes of Health and other funding agencies. For basic investigators: there would be opportunities to fine tune your research agenda. Again, not to change what you do, but to actually receive information that can help you to navigate the constant forks in the road that we all experience in our work. For clinical investigators, there will be the opportunity to have a greater application of your findings. And, for health services researchers, less risk of having to play "catch up" when new devel- opments of which you are unaware arrive from the research pipeline.

So what do I mean specifically? I mean that we need to develop research teams of basic, clinical and health services researchers; where basic and clinical researchers, with the help of health services researchers, are looking forward in the research continuum to implementation of their ideas, and that health services researchers are looking back, in the research continuum, with basic and clinical researchers to guide their prospective work.

Now for some specific examples of how this can work. Two of the fastest growing fields, and those with some of the shortest times from concept to products are genomics and proteomics. Because I do most of my own research on immunizations, I will use that topic as the template for these examples.

Exciting and groundbreaking research is being conducted in vaccine genomics, with efforts to determine genetic markers which predict the individual variation in the level of immune response to vaccination. The application of this work would be to determine which children may need additional doses or conversely, fewer doses, of vaccine to achieve an adequate immune response. Other work is looking for genetic markers to determine which children may have a higher likelihood of adverse reactions.

Both of these lines of research have tremendous potential. However, assessment of the manner by which such developments could be instituted into practice must also occur concurrently. Research into the potential cost of testing, where such testing could be conducted, the likelihood of payment in the public or private sector, the availability and interpretation of testing to different patient populations, and the potential for unintended consequences need to be assessed if such research will ever impact the lives of populations of children. By working together, investigators across the research continuum can help inform each other's research agenda, especially as the pace of new innovation increases. The basic work will inform the implementation studies, and the implementation studies will provide a "reality check" to help fine tune the direction of the technology development.

Developments in proteomics are resulting in an explosion of new vaccine candidates and an enlarging array of potentially preventable diseases. In this example, implementation research can help to guide which vaccine candidates should be pursued based on studies of the likelihood of a particular vaccine being used by providers and patients and paid for by public or private insurance.

But, there are some significant roadblocks to overcome if we are to change the way we think about the research continuum. Metaphorically, we need to figure out how porcupines mate; what we need to do to facilitate interaction and encourage cooperation. At the same time we have to face the issues of tribalism I mentioned earlier, we must confront our own arrogance that leads us believe some types of rigorously conducted research are more valuable than others. We must also address the structural roadblocks of our academic reward models that are struggling to recognize fairly the credit, both academic and financial, that accrues with team-based research, 
